\title{
Relevant Factors and Optimization in the Management of a PET/CT Facility
}

\author{
Antonio Orlacchio $^{1}$, Anna Micaela Ciarrapico ${ }^{2}$, Orazio Schillaci ${ }^{1}$, Manlio Guazzaroni ${ }^{1}$, \\ Tommaso Volpi ${ }^{1}$, Roberta Danieli ${ }^{1}$, Giovanni Simonetti ${ }^{1}$ \\ ${ }^{1}$ Department of Diagnostic and Molecular Imaging, Interventional Radiology, Nuclear Medicine and Radiation Therapy, \\ University Hospital “Tor Vergata”, Rome, Italy \\ ${ }^{2}$ Department of Public Health, University of Rome Tor Vergata, Rome, Italy \\ Email: aorlacchio@uniroma2.it
}

Received August 6, 2012; revised September 3, 2012; accepted September 15, 2012

\begin{abstract}
Objectives: To analyze the whole PET/CT imaging protocol from patient's registration to the end of the exam in order to optimize the use of such device. Methods: Data from June to July 2010 and from September to October 2010 were acquired, evaluating the time required by every step of the patient pathway. After the first data acquisition, we identified critical elements connected with the execution of the exams. Results: The results of the first data acquisition reported 12 daily performed exams. By reducing patient turnover time and consequently the device downtime, patient turnover time dropped from 6 min to only 3 min while device downtime dropped from $2 \mathrm{~h}$ and 30 min to $1 \mathrm{~h}$ and 20 min between the first and the second data collection. The number of daily performed exams increased from 12 to 14. Conclusions: The results show how the analysis of a complex study process such as PET/CT and a continuous activity control allow the identification of critical organizational and structural issues providing necessary information to the optimization in the use of devices with a clear value in public health, in large benefits for the patients and improved management results.
\end{abstract}

Keywords: Cost-Effectiveness; Positron Emission Tomography; Computerized Tomography; Image Fusion

\section{Introduction}

$\mathrm{PET} / \mathrm{CT}$ is a technology mainly used in cancer staging and with a therefore clear value in public health. Optimizing the use of such device, and the consequent opportunity to perform the highest possible number of exams during the device's operational hours, reduce waiting lists and make the resource management more effective [1-4].

In fact the maximum number of exams that can be performed during the department's operating hours is given by the combination of the device's operational hours and the acquisition protocol.

The aim of this study is the analysis of the whole PET/CT imaging protocol from the patient's registration to the end of the exam, paying particular attention to the time required for every step of the process, the number of examined patients and their stay in the department. Besides we analyzed critical points of the process to provide information of general interest.

\section{Materials and Method}

\subsection{Center Organization}

The study center's PET/CT is operational six days a week from Monday to Saturday: From 8 a.m. (registration time of the first patient) to 6 p.m. (end of the last exam) Monday till Friday, and 8 a.m. to 2 p.m. on Saturday. During operative hours, hospital representatives dedicated to patient registration, a Nuclear Medicine Physician, a Radiologist, a nurse and a Radiologic Technician are present in the department.

The reception desk is open from 8 a.m. to 7 p.m.

Regarding the radiopharmaceuticals produced by the cyclotron of the Hospital hosting the PET/CT center, a quantity of 18F-FDG proportional to the number of patients is delivered twice a day.

\subsection{The Patient Pathway}

After registration, the patient is interviewed by a physician in order to obtain information regarding his medical history, to verify the indications for the exam and to obtain written consent.

A nurse performs a glucose test and if the result is in the normal values range, the nuclear medicine physician endovenously injects 18F-FDG. If blood glucose values are higher than normal ones, the patient is asked to take a walk for 10 - 15 minutes before a further glucose test is performed. In the rare cases in which blood glucose level 
doesn't reach acceptable values, the exam is postponed.

The patient is then taken to a quiet and dimly lit room, isolated from the rest of the department, in which he is administered intravenous saline and oral contrast medium in order to highlight the bowel during the CT scan. 18F-FDG uptake requires 45 - 60 minutes after which the patient is requested to void the bladder, subsequently positioned into the PET/CT device by the radiologic technician and, upon the radiologist's indication, connected to the contrast fluid delivery system.

A non-contrast CT scan is performed providing attenuation correction for the subsequent PET scan and anatomic localization of FDG uptakes.

After the PET scan is over, the quality of the acquired images is evaluated and, when indicated, a second CT scan is performed with the use of contrast media injected by the radiologist.

Acquired images are then sent to an imaging workstation and jointly evaluated by the nuclear medicine physician and the radiologist who produce together a single medical report including morphologic, metabolic and functional information, and diagnostic conclusions. Furthermore, in the case of pathological findings, the most significant images are printed.

Meanwhile the radiologic technician burns a CD containing the complete exam, which will be given with the report.

\subsection{Relevant Elements for the Patient Pathway's Rationalization}

Figure 1 shows variables influencing the exam execution process with their relative weight. Weights represent the influence of the single factor not only in reference to the study Center. In the scheme, a weight of 3 or 4 is associated to crucial factors. As evidenced, considering the patient availability organized into a waiting list, and given the supposed availability in an organized center of radionuclids, nuclear medicine physicians and radiologists, the most important factors are patient punctuality, a space arrangement facilitating the patient pathway and the avoidance of eventual interferences with exam planning by emergencies.

Data collection was performed highlighting the most important elements in the center's organization and administering a questionnaire on such topics to patients, physicians, nurses and admitting representatives.

\subsection{Data Collection}

For the analysis, data from two periods were acquired: the first period from June to July 2010 and the second period from September to October 2010. During these periods questionnaires were administered in our PET/CT center of the Diagnostic Imaging department to administrative staff, nurses, radiologic technicians and physicians.

This approach allowed the evaluation of the time required by every single step of the patient pathway, from registration to the end of the exam.

After the first data acquisition, we identified the critical elements leading to the execution of only 12 exams a day despite the fact that the maximum number of exams which could be performed in the study PET/CT center was 14 .

The outpatient/inpatient ratio remained unchanged between the two data collections (Figure 2).

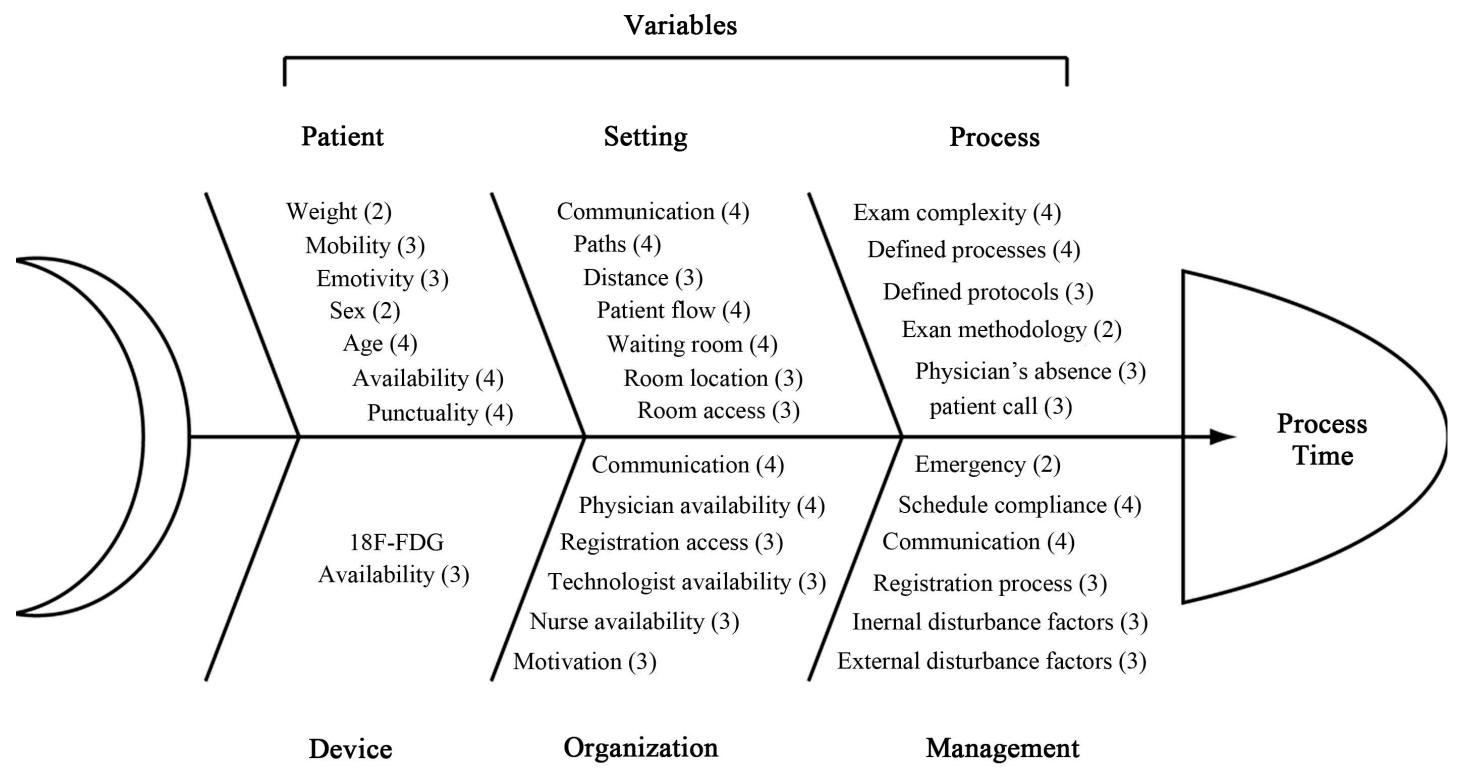

Figure 1. The fishbone diagram shows the factors influencing the examination process; 4 and 3 indicate the most important weight. 
First data collection period

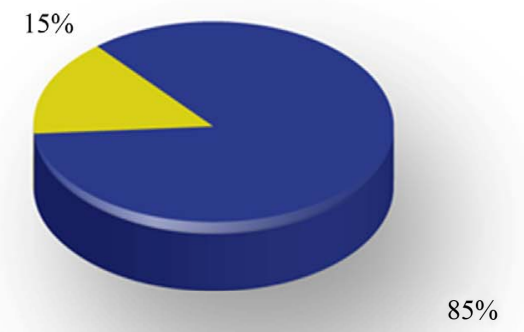

Second data collection period

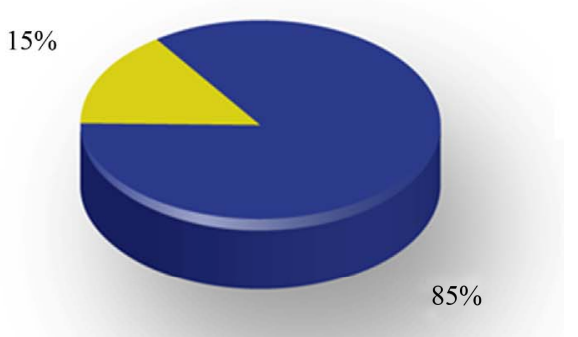

Figure 2. The diagrams shows the percentages of inpatient and outpatient in comparison between the two periods of data collection.

\section{Results}

The results of the first data acquisition, shown in Table 1, report 12 daily performed exams.

We therefore analyzed the device downtime and patient turnover time. This allowed a better organization which, by reducing patient turnover time and conesquently the device downtime, led to an increased use of the machine. Between the first and second data collection, patient turnover time dropped from 6 minutes to only 3 minutes while device downtime dropped from 2 hours and 30 minutes to 1 hour and 20 minutes. As a result, the number of daily performed exams increased from 12 to 14.

Regarding the total exam execution process time, from patient arrival in the department to the end of the exam, no significant change was recorded between the two data collection periods: As reported in Table 2 the average time was 165.5 during the first acquisition and 160.3 during the second one.

Analyzing data from the second collection period, total execution time was evaluated from the patients' scheduled appointment time as well. In this way the total required time decreased. This discrepancy could be explained by a certain variability in patient arrival time which causes a high standard variation.

Patient punctuality in both data collection periods was variable with most patients arriving early in the first data collection period while a very high rate of patients arrived late during the second one.

The mismatch between patient arrival and the scheduled time exceeded specifications in 53\% of the cases in the second period, and in $57.6 \%$ of the cases in the first period. During the second data collection period, $61 \%$ of the patients underwent the exam with an average delay of 30 minutes while during the first period an average 35 minutes delay occurred in $44 \%$ of the cases. During the first data collection period, $39 \%$ of the exams was per- formed on average 35 minutes in advance while, during the second period, $23 \%$ were carried out 40 minutes early on average.

A mismatch of over 10 minutes between the scheduled time and the actual time of the beginning of the exam process was recorded in $62 \%$ of the cases in the first period and in $69.5 \%$ in the second period.

As shown in Table 3, some steps in the exam execution process happened to be critical since they involved long waiting times.

Waiting times for the interview, which was the first step of the exam execution process, were calculated starting from both actual patient arrival time and from scheduled time. Interview waiting time had a 9.8\% (12.2\% if considered starting from the scheduled time) influence on the overall exam duration in the second data collection period compared to a $11.8 \%$ influence in the first one. Glucose test waiting time had a $19 \%$ influence on the overall PET/CT examination time in the second data collection period compared to a $15.2 \%$ influence in the first one. 18F-FDG administration waiting time had a $21.5 \%$ influence on the overall study time in the second data collection period compared to a $25.4 \%$ influence in the first one. Even though Glucose test waiting time had great impact on the overall exam execution time, it was not the single relevant factor as the decrease of the interview waiting time and 18F-FDG administration waiting time had led to improvements between the two data collections.

\section{Discussion}

The detailed analysis of the PET/CT study process and its single steps led to the identification of critical points which allowed a better organization of the patient path and to a consequent increased number of examinations performed between the first and second data collection. 
Table 1. Overall results.

\begin{tabular}{ccc}
\hline \multicolumn{3}{c}{ Length mins (SD) } \\
\hline & 1st Data Collection & 2nd Data Collection \\
\hline $\begin{array}{c}\text { Patients per day } \\
\begin{array}{c}\text { Average device } \\
\text { downtime }\end{array}\end{array}$ & 12 & 14 \\
$\begin{array}{c}\text { Device usage } \\
\text { Average } \\
\text { turnover time }\end{array}$ & $76 \%$ & $1 \mathrm{hr} 20 \mathrm{~min}$ \\
\hline
\end{tabular}

Table 2. Total study duration.

\begin{tabular}{ccc}
\hline \multicolumn{3}{c}{ Length min (SD) } \\
\hline & 1st Data Collection & 2nd Data Collection \\
\hline $\begin{array}{c}\text { Study length from patient } \\
\text { arrival } \\
\begin{array}{c}\text { Study length from } \\
\text { scheduled time }\end{array}\end{array}$ & $165.5(43)$ & $160.3(60)$ \\
\hline
\end{tabular}

Table 3. PET/CT study steps duration (see text for explanation).

\begin{tabular}{ccc}
\hline Steps & \multicolumn{2}{c}{ Length min } \\
\hline & 1st Data Collection & 2nd Data Collection \\
\hline Registration & 3.9 & 3.8 \\
Interview waiting time & 19.5 & 15.7 \\
Interview & 9 & 9 \\
Glucose test waiting time & 25.2 & 30.6 \\
FDG administration & 67.3 & 65 \\
waiting time & 3.5 & 3.7 \\
Patient preparation & 26 & 26 \\
Standard PET study & 11.1 & 6.5 \\
$\quad$ CT study & 165.5 & 160.3 \\
& & \\
\hline
\end{tabular}

It is important to highlight the fact that in literature the break even point for a PET/CT facility was estimated to be between 8 and 9 daily exams, a value confirmed by the management of the study center $[5,6]$. Therefore the number of performed procedures recorded during both data acquisition periods was above such break even point [7]. However, given the value in public health of PET/CT and the amount of the initial investment, the importance of executing the maximum possible number of exams is clear $[8,9]$.

In the study center, considering the patient pathway, the device's operational hours and the department's operative hours, the estimated maximum number of exams that can be performed daily, Monday through Friday, was 14 .
The results of the first data acquisition report 12 daily performed exams. We therefore analyzed the device downtime and patient turnover time. This allowed a better organization which, by reducing patient turnover time and consequently the device downtime, led to an increased use of the machine.

Between the first and second data collection, there was a $11.5 \%$ increase in the device usage rate while downtime remained equally balanced between morning and afternoon.

Average patient turnover time in the second data collection was 3 minutes, 3 minutes shorter than in the first data collection. This decreased turnover time showed that dead times were concentrated at the beginning and at the end of the day, and allowed the execution of an increased number of exams in the second analyzed period, with an increase from 12 to 14 exams.

Despite due to architectural limitations some steps in the patient's path could not be shortened and, in some cases, required longer times such as glucose test, the total lenght of the process decreased from 164.5 to $162.3 \mathrm{~min}$ utes, while standard deviation grew from 43 to $60 \mathrm{~min}$ utes.

Data from the second data collection showed that 23\% of the patients arrive, on average, 40 minutes early and that $61 \%$ of the patients underwent examination with an average delay of 30 minutes. Variability in patient arrival was likely due to unpredictable and uncorrectable factors such as traffic. In order to reduce dead times, the patient, should start the exam execution process as soon as possible from its arrival.

A further study could investigate whether punctuality in combination with a more organized and fluent path could reduce dead times and offer more benefits for the patients.

Therefore, further improvements in the scheduling process and in the management of the patient path have to be made taking account of uncorrectable factors in order to obtain greater punctuality in the PET/CT study execution and a more orderly flow with consequent benefits in terms of patient satisfaction.

Furthermore, an excessive patient delay may lead to limited availability of 18F-FDG which is delivered considering the decay time of the radionuclide and the scheduled examination time.

A further point of interest was the combination between the PET study and a diagnostic contrast CT examination. Considering data from both collection periods, in our experience the second one required 5 minutes of the total examination process time. Even though this may appear to be a lengthening of the PET/CT study, its derived benefits must be considered. The main advantage consists in the fact that patients (mainly those affected by oncological diseases) who would in any case have to 
undergo both PET and contrast-enhanced CT examinations, receive both in a single process, with a reduction in terms of time, costs and stress. This consequently leads to increased schedulable CT time and shorter CT waiting lists.

In order to obtain such a result, the main effort of our Center was to inform referring physicians and evaluate the clinical needs of the patients before the examination accurately.

In conclusion, the obtained results evidence how the thorough analysis of a complex study process such as PET/CT and a continuous activity control allow the identification of critical organizational and structural issues and therefore provide useful information to the optimization in the use of expensive and sophisticated devices with a clear value in public health, great benefits for the patients and improved management results.

\section{REFERENCES}

[1] A. K. Buck, K. Herrmann, T. Stargardt, T. Dechow, B. J. Krause and J. Schreyögg, "Economic Evaluation of PET and PET/CT in Oncology: Evidence and Methodologic Approaches,” Journal of Nuclear Medicine, Vol. 51, No. 3, 2010, pp. 401-412. doi:10.2967/jnumed.108.059584

[2] M. W. Saif, I. Tzannou, N. Makrilia and K. Syrigos, "Role and Cost Effectiveness of PET/CT in Management of Patients with Cancer," Yale Journal of Biology and Medicine, Vol. 83, No. 2, 2010, pp. 53-65.

[3] M. E. Juweid and B. D. Cheson, "Positron-Emission to- mography and Assessment of Cancer Therapy,” The New England Journal of Medicine, Vol. 354, No. 5, 2006, pp. 496-507. doi:10.1056/NEJMra050276

[4] A. Orlacchio, O. Schillaci, E. Gaspari, F. Della Gatta, R. Danieli, F. Bolacchi, C. Ragano Caracciolo, A. Mancini and G. Simonetti, "Role of $\left[{ }^{18} \mathrm{~F}\right]$-FDG-PET/MDCT in Evaluating Early Response in Patients with Hodgkin's Lymphoma," La Radiologia Medica, Vol. 117, No. 7, 2012, pp. 1250-1263. doi:10.1007/s11547-012-0792-8

[5] P. S. Conti, J. S. Keppler and J. M. Halls, "Positron Emission Tomography: A Financial and Operational Analysis,” American Journal of Roentgenology, Vol. 162, No. 6, 1994, pp. 1279-1286.

[6] J. S. Keppler and P. S. Conti, "A Cost Analysis of Positron Emission Tomography,” American Journal of Roentgenology, Vol. 177, No. 1, 2001, pp. 31-40.

[7] B. Krug, A. S. Pirson, R. Crott and T. Vander Borght, "Is a Methodology Available That Accurately Measures the Cost of an FDG-PET Study?” European Journal of $\mathrm{Nu}$ clear Medicine and Molecular Imaging, Vol. 34, No. 5, 2007, pp. 625-657. doi:10.1007/s00259-006-0308-y

[8] S. Halliday and J. H. Thrall, "The Business of PET/CT," American Journal of Roentgenology, Vol. 184, No. 5, 2005, pp. S152-S155.

[9] R. Remonnay, M. Morelle, P. Pommier, F. Giammarile and M. O. Carrere, “Assessing Short Term Effects and Costs at an Early Stage of Innovation: The Use of Positron Emission Tomography on Radiotherapy Treatment Decision Making,” International Journal of Technology Assessment in Health Care, Vol. 24, No. 2, 2008, pp. 212220. doi:10.1017/S026646230808029X 